\title{
A FULL INVESTIGATION OF THE DIRECTIONAL CONGESTION IN DATA ENVELOPMENT ANALYSIS
}

\author{
Somayeh Khezri ${ }^{1, *}$, Akram Dehnokhalaji² and Farhad Hosseinzadeh Lotfi ${ }^{3}$
}

\begin{abstract}
One of interesting subjects in Data Envelopment Analysis (DEA) is estimation of congestion of Decision Making Units (DMUs). Congestion is evidenced when decreases (increases) in some inputs result in increases (decreases) in some outputs without worsening (improving) any other input/output. Most of the existing methods for measuring the congestion of DMUs utilize the traditional definition of congestion and assume that inputs and outputs change with the same proportion. Therefore, the important question that arises is whether congestion will occur or not if the decision maker (DM) increases or decreases the inputs dis-proportionally. This means that, the traditional definition of congestion in DEA may be unable to measure the congestion of units with multiple inputs and outputs. This paper focuses on the directional congestion and proposes methods for recognizing the directional congestion using DEA models. To do this, we consider two different scenarios: (i) just the input direction is available. (ii) none of the input and output directions are available. For each scenario, we propose a method consists in systems of inequalities or linear programming problems for estimation of the directional congestion. The validity of the proposed methods are demonstrated utilizing two numerical examples.
\end{abstract}

Mathematics Subject Classification. 90C05, 90C29, 90B50.

Received May 18, 2018. Accepted September 18, 2019.

\section{INTRODUCTION}

In recent years, DEA has been applied as a powerful tool for performance evaluation. Since then, a large amount of studies has been done in DEA theory and practice. Emrouznejad and Yang [9] reported DEA studies from 1978 to end of 2016. The concept of congestion is an important subject in DEA and management. It occurs when the reduction (increment) in some inputs results in the maximum possible increase (decrease) in some outputs without worsening (improving) other input/output [7]. The DM can apply congestion to decide about increasing or decreasing the size of a particular DMU. The problem of the congestion estimation of decision making units has attracted attentions of several scholars. Färe and Sevensson [10] formulated a linear programming model to define the concept of congestion in a production technology with a single output. Färe et al. [11] emphasized on the efficiency evaluation and proposed a radial model to estimate the congestion.

Keywords. Data envelopment analysis (DEA), directional congestion, decision making units.

1 Department of Mathematics, Faculty of Mathematical Sciences and Computer, Kharazmi University, Tehran, Iran.

2 Operations and Information Management Department, Aston Business School, B4 7ET Birmingham, UK.

3 Department of Mathematics, Science and Research Branch, Islamic Azad University, Tehran, Iran.

*Corresponding author: khezri_s@ut.ac.ir 
Because of the congestion is a kind of inefficiency, therefore, their model may be unable to recognize the congestion in some situations. Later, Cooper et al. [3] proposed a slack-based method to measure the congestion of DMUs. The advantage of their method over previous methods is that it not only distinguishes the congested inputs, but also measures the amount of congestion of each input. Cooper et al. [4] developed an additive model for identifying the congestion of units.

Regarding the traditional definition of congestion, the congestion is evidenced when the increase in some inputs results in the decrease in some outputs, hence, it can identify the shortfall of outputs. In this respect, Wei and Yan [23] and Tone and Sahoo [21] considered the congestion in outputs. Wei and Yan [24] considered the output oriented DEA models to recognize the necessary and sufficient conditions for the existence of congestion. Sueyoshi and Sekitani [20] dealt with the situation that there exist alternative optimal solutions in models for measuring the congestion and proposed an approach to measure the congestion of decision making units. Ghomashi and Abbasi [12] proposed a linear inequality and equality system to estimate the congestion of decision making units. Kheirollahi et al. [15] developed an input relaxation model to identify the input congestion of units in data envelopment analysis with fuzzy data. Khoveyni et al. [16] proposed mixed integer programming (MIP) models to determine the strongly and weakly most congested units in the presence of negative data. Mehdiloozad et al. [18] introduced the concept of Max-projection for the congestion of inefficient units and proposed a linear programming model to identify the Max-projection and also developed a single-stage LP model to estimate the congestion of units. Ebrahimzade Adimi et al. [8] introduced the concept of the congestion hyperplane and applied it to measure the congestion of DMUs. For more studies about congestion see Jahanshahloo and Khodabakhshi [13], Kao [14], Noura et al. [19], Khoveyni et al. [17] and Wu et al. [25-27].

The conventional DEA models assume that all inputs and outputs are deterministic. However, this assumption may not always hold true due to existence of uncertainty for example inputs and outputs might be stochastic or fuzzy data. See Wanke et al. [22] for more studies about the comparison between DEA and fuzzy DEA models.

There are two basic terms in the congestion literature: strong and weak congestion. If decreases in all (some) inputs of the DMU result in increases in all (some) outputs of it, then this decision making unit has strong (weak) congestion. There exist main drawbacks with the definitions of strong and weak congestion. First, strong and weak congestion only consider the situation that the decreases in inputs can be associated with the increases in outputs and the case that the increases in some inputs result in the decreases in some outputs without improving any other input or output is not considered in these definitions. Second, in case of strong or weak congestion, we cannot recognize the precise direction along which the congestion occurs. This means that we do not know whether congestion will occur or not if the DM increases (or decreases) inputs and outputs disproportionally. Therefore, we may be unable to estimate the congestion of DMUs with multiple inputs and outputs. In this regard, Yang [28] proposed the definition of the directional congestion along certain input and output directions. He proposed two methods with different perspectives to estimate the directional congestion. Both methods assume that input and output directions are predetermined by the DM.

The methods of Yang [28] only consider the existence of congestion along the certain input/output directions. In other words, if a DMU has no congestion along these certain directions, no information about the existence or absence of the congestion along other directions can be obtained. Therefore, we consider the situation in which at least one input or output direction is not specified. Two different scenarios are considered in this paper: (i) a scenario in which only the input direction is specified. We present two methods to estimate the directional congestion for this scenario. If there exists the congestion along a certain input direction, both methods can find an output direction along which congestion occurs. (ii) a scenario based on the assumption that both input and output directions are not specified. We propose a system of inequalities to find input and output directions along which the congestion occurs in this scenario. This study addresses the relationship between our defined directional congestion and the classical definition of strong and weak congestion.

The rest of this paper is organized as follows: Section 2 reviews preliminaries and basic definitions. In Section 3 , we present three methods to estimate the directional congestion. Numerical examples are provided in Section 4. Section 5 concludes the paper. 


\section{Preliminaries And BAsic Definitions}

Suppose that there exist $n$ decision making units, $\mathrm{DMU}_{j}, j=1, \ldots, n$, and each DMU consumes $m$ inputs to produce $s$ outputs. The ith input and $r$ th output for $\mathrm{DMU}_{j}$ are denoted by $x_{i j}$ and $y_{r j}$, respectively for $i=1, \ldots m$ and $r=1, \ldots, s$. We assume that all input and output values are non-negative, and at least one of each is non-zero. Let $\mathrm{DMU}_{o}=\left(x_{o}, y_{o}\right)$ be the unit under assessment. The production Possibility Set (PPS) with variable returns to scale (VRS) defined by Banker et al. [1] is as follows:

$$
T_{v}=\left\{(x, y) \mid x \geq \sum_{j=1}^{n} \lambda_{j} x_{j}, y \leq \sum_{j=1}^{n} \lambda_{j} y_{j}, \sum_{j=1}^{n} \lambda_{j}=1, \lambda_{j} \geq 0, j=1, \ldots, n\right\} .
$$

The output-oriented BCC model proposed by Banker et al. [1] for evaluating the efficiency score of $\mathrm{DMU}_{o}$ is as follows:

$$
\begin{aligned}
& \psi^{*}=\max \rho+\epsilon\left(\sum_{i=1}^{m} s_{i}^{-}+\sum_{r=1}^{s} s_{r}^{+}\right) \\
& \text {s.t. } \sum_{j=1}^{n} \lambda_{j} x_{i j}+s_{i}^{-}=x_{i o}, \quad i=1, \ldots, m, \\
& \sum_{j=1}^{n} \lambda_{j} y_{r j}-s_{r}^{+}=\rho y_{r o}, \quad r=1, \ldots, s, \\
& \sum_{j=1}^{n} \lambda_{j}=1 \\
& \lambda_{j} \geq 0 \text {, } \\
& s_{i}^{-} \geq 0 \text {, } \\
& s_{r}^{+} \geq 0 \text {, } \\
& \begin{array}{l}
j=1, \ldots, n \\
i=1, \ldots, m \\
r=1, \ldots, s
\end{array}
\end{aligned}
$$

where $\epsilon$ is non-Archimedean.

Banker et al. [1] presented the following definition:

Definition 2.1. Suppose that $\left(\rho^{*}, s^{-^{*}}, s^{+^{*}}, \lambda^{*}\right)$ is an optimal solution for model (2.2) evaluating $\mathrm{DMU}_{o}$. If $\rho^{*}=1$ then $\mathrm{DMU}_{o}$ is called technically efficient. Furthermore, if $\psi^{*}=1, \mathrm{DMU}_{o}$ is called strongly efficient.

Cooper et al. [5] and Brockett et al. [2] presented the classical definition of congestion as follows:

Definition 2.2. The unit $\mathrm{DMU}_{o}=\left(x_{o}, y_{o}\right)$ has congestion if the decreases (increases) in some inputs result in the increases (decreases) in some outputs without worsening (improving) other inputs/outputs.

There are several approaches to recognize the congestion. For example, Tone and Sahoo [21] proposed TS method which is summarized as follows:

\subsection{TS method}

Tone and Sahoo [21] defined the PPS accepting all assumptions to build $T_{v}$ except one assumption, strong disposal. They considered weak disposal instead, which was defined as follows:

Definition 2.3. The PPS satisfies weak disposal assumption if for each $(\bar{x}, \bar{y})$ belonging to the PPS and vector $(x, y)$ where $x=\bar{x}$ and $y \leq \bar{y},(x, y)$ belongs to the PPS. 
Therefore, Tone and Sahoo [21] presented the following PPS:

$$
P_{\text {convex }}=\left\{(x, y) \mid x=\sum_{j=1}^{n} \lambda_{j} x_{j}, y \leq \sum_{j=1}^{n} \lambda_{j} y_{j}, \sum_{j=1}^{n} \lambda_{j}=1, \lambda_{j} \geq 0, j=1, \ldots, n\right\} .
$$

They proposed the following model to evaluate the efficiency score of $\mathrm{DMU}_{o}$, with respect to $P_{\text {convex }}$ :

$$
\begin{aligned}
& \Phi^{*}=\max \Phi+\epsilon\left(\sum_{r=1}^{s} s_{r}^{+}\right) \\
& \text {s.t. } \sum_{j=1}^{n} \lambda_{j} x_{i j}=x_{i o}, \quad i=1, \ldots, m, \\
& \sum_{j=1}^{n} \lambda_{j} y_{r j}-s_{r}^{+}=\Phi y_{r o}, \quad r=1, \ldots, s, \\
& \sum_{j=1}^{n} \lambda_{j}=1 \\
& \lambda_{j} \geq 0 \text {, } \\
& j=1, \ldots, n, \\
& s_{r}^{+} \geq 0 \text {, } \\
& r=1, \ldots, s \text {. }
\end{aligned}
$$

It is clear that the target unit for $\mathrm{DMU}_{o}$, located on the strongly efficient frontier of $P_{\text {convex }}$ is as follows:

$$
\begin{array}{ll}
\hat{x}_{i o}=x_{i o}, & i=1, \ldots, m, \\
\hat{y}_{r o}=\Phi^{*} y_{r o}+s_{r}^{+*}, & r=1, \ldots, s .
\end{array}
$$

Tone and Sahoo [21] presented the following definitions for strongly efficient unit, strong congestion and weak congestion, respectively:

Definition 2.4. The unit $\mathrm{DMU}_{o}=\left(x_{o}, y_{o}\right)$ is strongly efficient unit with respect to $P_{\text {convex }}$, if $\Phi^{*}=1$.

Definition 2.5. Suppose that $\mathrm{DMU}_{o}=\left(x_{o}, y_{o}\right)$ is strongly efficient unit with respect to $P_{\text {convex }}$. $\mathrm{DMU}_{o}$ has strong congestion if there exists $\left(\bar{x}_{o}, \bar{y}_{o}\right) \in P_{\text {convex such that }} \bar{x}_{o}=\alpha x_{o}(0<\alpha<1)$ and $\bar{y}_{o} \geq \beta y_{o}(\beta>1)$.

Definition 2.6. Suppose that $\mathrm{DMU}_{o}=\left(x_{o}, y_{o}\right)$ is strongly efficient unit with respect to $P_{\text {convex }}$. $\mathrm{DMU}_{o}$ has weak congestion if there exists an activity in $P_{\text {convex }}$ that uses less resources in some components of the input vector to produce more products in some components of the output vector.

Tone and Sahoo [21] assumed that $\mathrm{DMU}_{o}$ is strongly efficient unit with respect to $P_{\text {convex }}$. If not, they projected $\mathrm{DMU}_{o}$ on to the strongly efficient frontier of $P_{\text {convex }}$ and then applied the following method to recognize the strongly and weakly congested units:

Step 1. Solve model (2.2). Suppose that $\left(\rho^{*}, s^{-*}, s^{+*}, \lambda^{*}\right)$ is an optimal solution for this model:

(a) If $\rho^{*}=1, s^{-*}=0, s^{+*}=0$, then $\mathrm{DMU}_{o}=\left(x_{o}, y_{o}\right)$ is BCC-efficient and not congested.

(b) If $\rho^{*}=1, s^{-*} \neq 0, s^{+*}=0$, then $\mathrm{DMU}_{o}=\left(x_{o}, y_{o}\right)$ is technically inefficient.

(c) If $\rho^{*}=1, s^{+*} \neq 0$ or $\rho^{*}>1$, then $\operatorname{DMU}_{o}=\left(x_{o}, y_{o}\right)$ has congestion. 
Go Step 2.

Step 2. Solve model (2.6):

$$
\begin{array}{ll} 
& \tilde{u}=\max u_{o} \\
\text { s.t. } & \sum_{r=1}^{s} u_{r} y_{r o}=1 \\
& \sum_{r=1}^{s} u_{r} y_{r j}-\sum_{i=1}^{m} v_{i} x_{i j}+u_{o} \leq 0, \\
& \sum_{r=1}^{s} u_{r} y_{r o}-\sum_{i=1}^{m} v_{i} x_{i o}+u_{o}=0, \\
& u_{r} \geq 0 \\
& v_{i} \text { free } \\
& u_{o} \text { free. }
\end{array}
$$

suppose that $\tilde{u}$ is the optimal value of model (2.6) and $\tilde{\rho}=1+\tilde{u}$. If $\tilde{\rho}<0$ then $\mathrm{DMU}_{o}$ has strong congestion, otherwise it has weak congestion.

Step 3. Termination.

The definition of strong and weak congestion has main drawbacks. First, strong and weak congestion only consider the situation that decreases in inputs can be associated with increases in outputs and the case that increases in inputs result in decreases in outputs without improving any other input or output, is not considered in these definitions. Second, in case of strong or weak congestion, we cannot recognize the precise direction along which the congestion occurs. This means that we do not know whether congestion will occur or not if the DM increases (or decreases) inputs dis-proportionally. Therefore, we may be unable to estimate the congestion of DMUs with multiple inputs and outputs. In this regard, Yang [28] proposed the definition of directional congestion along certain input and output directions. He proposed two methods with different perspectives to estimate the directional congestion. Both methods assume that input and output directions are predetermined.

The methods of Yang [28] only consider the existence of congestion along the certain input/output directions. In other words, if a DMU has no congestion along these certain directions, no information about the existence or absence of the congestion along other directions can be obtained. Therefore, we consider the situation in which at least one input or output direction is not specified. Two different scenarios are considered in this paper: (i) a scenario in which only the input direction is specified. We present two methods to estimate the directional congestion for this scenario. If there exists the congestion along a certain input direction, both methods can find an output direction along which congestion occurs. (ii) a scenario based on the assumption that both input and output directions are not specified. We propose a system of inequalities to find input and output directions along which the congestion occurs in this scenario. This study addresses the relationship between our defined directional congestion and the classical definition of strong and weak congestion.

\section{OuR PROPOSED METhods FOR DETERMINING THE DIRECTIONAL CONGESTION}

In this section, we consider two different scenarios to recognize the directional congestion. In the first scenario only the input direction is specified. We present two methods to estimate the directional congestion for this scenario. One of them is based on solving linear programming problems and the other one is based on solving systems of inequalities. If a unit is directionally congested along a certain input direction, then our methods find an output direction along which congestion occurs. In the second scenario both input and output directions are not specified. We propose systems of inequalities to find the input and output directions along which congestion occurs in this scenario. 
We consider $P_{\text {convex }}$ in both scenarios and we investigate the congestion for the strong efficient unit with respect to $P_{\text {convex }}$.

\subsection{The first scenario}

Assume that the input direction vector $\vec{w}=\left(w_{1}, w_{2}, \ldots, w_{m}\right) \geq 0$ is specified by the DM along which the components of the input vector of $\mathrm{DMU}_{o}$ should be changed. First, the components of the input vector of $\mathrm{DMU}_{o}$ are decreased along $\vec{w}$ and we find an output direction vector $\left(\overrightarrow{\delta_{o}}\right)$ along which the output components of $\mathrm{DMU}_{o}$ are increased. If there exists such an output direction vector, then $\mathrm{DMU}_{o}$ is directionally congested from the left along $\vec{w}$. Then, the components of the input vector of $\mathrm{DMU}_{o}$ are increased along $\vec{w}$ and we find an output direction vector $\left(\overrightarrow{\delta_{o}^{\prime}}\right)$ along which the output components of $\mathrm{DMU}_{o}$ are decreased. If there exists such an output direction vector, then $\mathrm{DMU}_{o}$ is directionally congested from the right along $\vec{w}$. If $\mathrm{DMU}_{o}$ is directionally congested from the left and right, then this unit is directionally congested along $\vec{w}$. Otherwise $\mathrm{DMU}_{o}$ is not directionally congested along the input direction vector $\vec{w}$.

We propose two methods in Sections 3.1.1 and 3.1.2 to determine whether $\mathrm{DMU}_{o}$ is directionally congested along $\vec{w}$ or not.

In the first proposed method for the first scenario, we determine the output direction vectors along which the congestion occurs from the left and right by solving the systems of inequalities. In the second proposed method for the first scenario, we determine the output direction vectors along which the congestion occurs from the left and right by solving linear programming problems.

\subsubsection{The first method for the first scenario}

In this method, firstly, we consider the reduction in the components of the input vector of $\mathrm{DMU}_{o}$ along $\vec{w}$ and the increment in the components of the output vector of $\mathrm{DMU}_{o}$ along an output direction vector $\left(\overrightarrow{\delta_{o}}\right)$ obtained by solving a system of inequalities. Afterwards, we consider the increment in the components of the input vector of $\mathrm{DMU}_{o}$ along $\vec{w}$ and the reduction in the components of the output vector of this unit along an output direction vector $\left(\overrightarrow{\delta_{o}^{\prime}}\right)$ obtained by solving another system of inequalities. In the following, we present Algorithm I to recognize the directional congestion in the first scenario.

\section{Algorithm I}

Step 1. Solve model (3.1) to know whether we can decrease the components of the input vector of $\mathrm{DMU}_{o}$, along the input direction vector $\vec{w}=\left(w_{1}, w_{2}, \ldots, w_{m}\right)$ or not.

$$
\begin{aligned}
& \theta_{o}^{*}=\max \theta \\
& \text { s.t. } \sum_{j=1}^{n} \lambda_{j} x_{i j}=x_{i o}-\theta w_{i} x_{i o}, \quad i=1, \ldots, m \text {, } \\
& \sum_{j=1}^{n} \lambda_{j} y_{r j} \geq(1+\beta) y_{r o}, \quad r=1, \ldots, s, \\
& \sum_{j=1}^{n} \lambda_{j}=1 \\
& \lambda_{j} \geq 0, \quad j=1, \ldots, n, \\
& \theta \geq 0, \quad \quad \beta \text { free }
\end{aligned}
$$

Theorem 3.1. If $\theta_{o}^{*}=0$ at the optimality of model (3.1), then $\mathrm{DMU}_{o}$ is not directionally congested along $\vec{w}=\left(w_{1}, w_{2}, \ldots, w_{m}\right)$. 
Proof. If $\theta_{o}^{*}=0$, then the maximum possible decrease in the components of the input vector of $\mathrm{DMU}_{o}$ along $\vec{w}=\left(w_{1}, w_{2}, \ldots, w_{m}\right)$ is zero. This means that we cannot decrease inputs along $\vec{w}$. Hence, $\mathrm{DMU}_{o}$ is not directionally congested from the left along $\vec{w}$. Therefore, this unit is not directionally congested along $\vec{w}=\left(w_{1}, w_{2}, \ldots, w_{m}\right)$.

If $\theta_{o}^{*}>0$, then go to step 2 .

Step 2. Consider the following system of inequalities:

$$
\begin{array}{ll}
\sum_{j=1}^{n} \lambda_{j} x_{i j}=x_{i o}-\alpha w_{i} x_{i o}, & i=1, \ldots, m, \\
\sum_{j=1}^{n} \lambda_{j} y_{r j} \geq y_{r o}+\delta_{r o} y_{r o}, & r=1, \ldots, s \\
\sum_{j=1}^{n} \lambda_{j}=1, & \\
\sum_{r=1}^{s} \delta_{r o}=1, & \\
\lambda_{j} \geq 0, & \\
\delta_{r o} \geq 0, & \\
0 \leq \alpha \leq \theta_{o}^{*} . & r=1, \ldots, n, s,
\end{array}
$$

If system (3.2) has no solution, then $\mathrm{DMU}_{o}$ is not directionally congested from the left along $\vec{w}=$ $\left(w_{1}, w_{2}, \ldots, w_{m}\right)$ because there exists no direction vector along which the output components of $\mathrm{DMU}_{o}$ increase. Otherwise, there exists an output direction vector $\overrightarrow{\delta_{o}}=\left(\delta_{1 o}, \delta_{2 o}, \ldots, \delta_{s o}\right)$, obtained from system (3.2), such that the output components of $\mathrm{DMU}_{o}$ increase along it. This means that $\mathrm{DMU}_{o}$ is directionally congested from the left along the input direction $\vec{w}=\left(w_{1}, w_{2}, \ldots, w_{m}\right)$ and the output direction $\overrightarrow{\delta_{o}}=\left(\delta_{1 o}, \delta_{2 o}, \ldots, \delta_{s o}\right)$.

Go step 3 to determine the right-hand congestion:

Step 3. Solve model (3.3) to determine whether we can increase the components of the input vector of $\mathrm{DMU}_{o}$, along the input direction vector $\vec{w}=\left(w_{1}, w_{2}, \ldots, w_{m}\right)$ or not.

$$
\begin{aligned}
& \theta_{o}^{\prime *}=\max \theta^{\prime} \\
& \text { s.t. } \sum_{j=1}^{n} \lambda_{j} x_{i j}=x_{i o}+\theta^{\prime} w_{i} x_{i o}, \quad i=1, \ldots, m, \\
& \sum_{j=1}^{n} \lambda_{j} y_{r j} \geq(1+\beta) y_{r o}, \quad r=1, \ldots, s, \\
& \sum_{j=1}^{n} \lambda_{j}=1, \\
& \lambda_{j} \geq 0 \text {, } \\
& \theta^{\prime} \geq 0 \text {, } \\
& j=1, \ldots, n \text {, } \\
& \beta \text { free. }
\end{aligned}
$$

Theorem 3.2. If $\theta_{o}^{*}=0$ at the optimality of model (3.3), then $\mathrm{DMU}_{o}$ is not directionally congested along $\vec{w}=\left(w_{1}, w_{2}, \ldots, w_{m}\right)$. 
Proof. If $\theta_{o}^{\prime *}=0$ then the maximum possible increase in the components of the input vector of $\mathrm{DMU}_{o}$ along $\vec{w}=\left(w_{1}, w_{2}, \ldots, w_{m}\right)$ is zero. This means that we cannot increase inputs along $\vec{w}$. Hence, $\mathrm{DMU}_{o}$ is not directionally congested from the right along $\vec{w}$. Therefore, this unit is not directionally congested along $\vec{w}=\left(w_{1}, w_{2}, \ldots, w_{m}\right)$.

If $\theta_{o}^{\prime *}>0$, then go step 4 .

Step 4. Consider the following system of inequalities:

$$
\begin{array}{ll}
\sum_{j=1}^{n} \lambda_{j} x_{i j}=x_{i o}+\alpha w_{i} x_{i o}, & i=1, \ldots, m \\
\sum_{j=1}^{n} \lambda_{j} y_{r j} \geq y_{r o}-\delta_{r o}^{\prime} y_{r o}, & r=1, \ldots, s \\
\sum_{j=1}^{n} \lambda_{j}=1, & \\
\sum_{r=1}^{s} \delta_{r o}^{\prime}=1, & \\
\lambda_{j} \geq 0, & \\
\delta_{r o}^{\prime} \geq 0, & r=1, \ldots, n \\
0 \leq \alpha \leq \theta_{o}^{*} . &
\end{array}
$$

If system (3.4) has no solution, then $\mathrm{DMU}_{o}$ is not directionally congested from the right along $\vec{w}=$ $\left(w_{1}, w_{2}, \ldots, w_{m}\right)$ because there exists no direction vector along which the output components of $\mathrm{DMU}_{o}$ decrease. Otherwise, there exists an output direction vector $\overrightarrow{\delta_{o}^{\prime}}=\left(\delta_{1 o}^{\prime}, \delta_{2 o}^{\prime}, \ldots, \delta_{s o}^{\prime}\right)$, obtained from system (3.4), such that the output components of $\mathrm{DMU}_{o}$ decrease along it. This means that $\mathrm{DMU}_{o}$ is directionally congested from the right along the input direction $\vec{w}=\left(w_{1}, w_{2}, \ldots, w_{m}\right)$ and the output direction vector $\overrightarrow{\delta_{o}^{\prime}}=\left(\delta_{1 o}^{\prime}, \delta_{2 o}^{\prime}, \ldots, \delta_{s o}^{\prime}\right)$. If $\mathrm{DMU}_{o}$ is directionally congested from the both sides, then, this unit is directionally congested along the input direction $\vec{w}=\left(w_{1}, w_{2}, \ldots, w_{m}\right)$.

In what follows, we propose the second method.

\subsubsection{The second method for the first scenario}

In the following, we present Algorithm II to recognize the directional congestion in the first scenario.

\section{Algorithm II}

Step 1. Solve model (3.1) to determine whether we can decrease the components of the input vector of $\mathrm{DMU}_{o}$, along the input direction vector $\vec{w}=\left(w_{1}, w_{2}, \ldots, w_{m}\right)$ or not. As we said in Algorithm I, if $\theta_{o}^{*}=0$ at the optimality of model (3.1), then $\mathrm{DMU}_{o}$ is not directionally congested from the left along $\vec{w}=\left(w_{1}, w_{2}, \ldots, w_{m}\right)$. Otherwise, go to step 2.

Step 2. Solve model (3.5) or the linearized form of it, namely model (3.6), to find the output direction vector $\overrightarrow{\delta_{o}}=\left(\delta_{1 o}, \ldots, \delta_{s o}\right)$ along which the output components of $\mathrm{DMU}_{o}$ have the maximum possible increase:

$$
\begin{array}{ll}
\tau_{o}^{*}=\max \left(\min _{1 \leq r \leq s} \delta_{r o}\right) & \\
\text { s.t. } & \sum_{j=1}^{n} \lambda_{j} x_{i j}=x_{i o}-\alpha w_{i} x_{i o},
\end{array}
$$




$$
\begin{array}{ll}
\sum_{j=1}^{n} \lambda_{j} y_{r j} \geq y_{r o}+\delta_{r o} y_{r o}, & r=1, \ldots, s, \\
\sum_{j=1}^{n} \lambda_{j}=1, & \\
\sum_{r=1}^{s} \delta_{r o}=1, & \\
\lambda_{j} \geq 0, & j=1, \ldots, n, \\
\delta_{r o} \geq 0, & r=1, \ldots, s, \\
0 \leq \alpha \leq \theta_{o}^{*} . &
\end{array}
$$

Model (3.5) can be transformed into linear programming model (3.6) by introducing the variable $\tau=$ $\min _{1 \leq r \leq s} \delta_{r o}$ :

$$
\begin{aligned}
& \tau_{o}^{*}=\max \tau \\
& \text { s.t. } \sum_{j=1}^{n} \lambda_{j} x_{i j}=x_{i o}-\alpha w_{i} x_{i o}, \quad i=1, \ldots, m \text {, } \\
& \sum_{j=1}^{n} \lambda_{j} y_{r j} \geq y_{r o}+\delta_{r o} y_{r o}, \quad r=1, \ldots, s, \\
& \sum_{j=1}^{n} \lambda_{j}=1 \\
& \sum_{r=1}^{s} \delta_{r o}=1 \\
& \tau \leq \delta_{\text {ro }} \\
& \lambda_{j} \geq 0 \\
& \delta_{\text {ro }} \geq 0 \text {, } \\
& \tau \geq 0 \text {, } \\
& 0 \leq \alpha \leq \theta_{o}^{*} \text {. }
\end{aligned}
$$

The feasible set of model (3.6) is as follows:

$$
\begin{aligned}
S_{1}=\left\{\left(\alpha, \tau, \vec{\lambda}, \overrightarrow{\delta_{o}}\right) \mid \vec{\lambda} X \leq x_{o}-\alpha \vec{w} x_{o}, \vec{\lambda} Y \geq y_{o}+\overrightarrow{\delta_{o}} y_{o}, \overrightarrow{1} \cdot \vec{\lambda}=1, \overrightarrow{1} \cdot \overrightarrow{\delta_{o}}=1, \vec{\lambda} \in\right. \\
\left.\quad R_{\geq}^{n}, \overrightarrow{\delta_{o}} \in R_{\geq}^{s}, \alpha \leq \theta_{o}^{*}, \vec{\tau} \leq \overrightarrow{\delta_{o}}\right\} .
\end{aligned}
$$

where $\vec{\tau}=(\tau, \ldots, \tau) \in R^{s}$ and also $X=\left[x_{1}, x_{2}, \ldots, x_{n}\right]_{m \times n}$ and $Y=\left[y_{1}, y_{2}, \ldots, y_{n}\right]_{s \times n}$ are input and output matrices, respectively.

Therefore, there are two the following cases:

(1) If $S_{1}=\emptyset$, then there exists no output direction vector along which the components of the output vector of $\mathrm{DMU}_{o}$ increase. Therefore $\mathrm{DMU}_{o}$ is not directionally congested from the left along $\vec{w}=\left(w_{1}, w_{2}, \ldots, w_{m}\right)$, therefore it is not directionally congested along $\vec{w}$. 
(2) Let $S_{1} \neq \emptyset$ and $\left(\alpha^{*}, \tau^{*}, \overrightarrow{\lambda^{*}}, \overrightarrow{\delta_{o}^{*}}\right)$ be an optimal solution for model $(3.6)$. Therefore $\left(\delta_{1 o}^{*}, \delta_{2 o}^{*}, \ldots, \delta_{s o}^{*}\right)$ is the output direction vector along which the components of the output vector of $\mathrm{DMU}_{o}$ have the maximum possible increase. This means that $\mathrm{DMU}_{o}$ is directionally congested from the left along $\vec{w}=\left(w_{1}, w_{2}, \ldots, w_{m}\right)$, and go to step 3.

Step 3. Solve model (3.3) to determine whether we can increase the components of the input vector of $\mathrm{DMU}_{o}$, along the input direction vector $\vec{w}=\left(w_{1}, w_{2}, \ldots, w_{m}\right)$ or not. As we said in Algorithm I, if $\theta_{o}^{\prime *}=0$ at the optimality of model (3.3), then $\mathrm{DMU}_{o}$ is not directionally congested from the right along $\vec{w}=\left(w_{1}, w_{2}, \ldots, w_{m}\right)$. Otherwise, go to step 4.

Step 4. Solve model (3.8) or the linearized form of it, namely model (3.9), to find the output direction vector $\overrightarrow{\delta_{o}^{\prime}}=\left(\delta_{1 o}^{\prime}, \ldots, \delta_{s o}^{\prime}\right)$ along which the output components of $\mathrm{DMU}_{o}$ have the maximum possible decrease:

$$
\begin{aligned}
& \tau_{o}^{*}=\max \left(\min _{1 \leq r \leq s} \delta_{r o}^{\prime}\right) \\
& \text { s.t. } \sum_{j=1}^{n} \lambda_{j} x_{i j}=x_{i o}+\alpha w_{i} x_{i o}, \quad i=1, \ldots, m, \\
& \sum_{j=1}^{n} \lambda_{j} y_{r j} \geq y_{\text {ro }}-\delta_{\text {ro }}^{\prime} y_{\text {ro }}, \quad r=1, \ldots, s, \\
& \sum_{j=1}^{n} \lambda_{j}=1 \\
& \sum_{r=1}^{s} \delta_{r o}^{\prime}=1 \\
& \lambda_{j} \geq 0 \text {, } \\
& \delta_{r_{O}}^{\prime} \geq 0 \\
& j=1, \ldots, n, \\
& 0 \leq \alpha \leq \theta_{o}^{*} \text {. } \\
& r=1, \ldots, s \text {, }
\end{aligned}
$$

Model (3.8) can be transformed into linear programming model (3.9) by introducing the variable $\tau^{\prime}=$ $\min _{1 \leq r \leq s} \delta_{r o}^{\prime}:$

$$
\begin{aligned}
& \tau_{o}^{\prime *}=\max \tau^{\prime} \\
& \text { s.t. } \sum_{j=1}^{n} \lambda_{j} x_{i j}=x_{i o}+\alpha w_{i} x_{i o}, \quad i=1, \ldots, m \text {, } \\
& \sum_{j=1}^{n} \lambda_{j} y_{r j} \geq y_{r o}-\delta_{r o}^{\prime} y_{r o}, \quad r=1, \ldots, s, \\
& \sum_{j=1}^{n} \lambda_{j}=1 \\
& \sum_{r=1}^{s} \delta_{r o}^{\prime}=1 \\
& \tau^{\prime} \leq \delta_{\text {ro }}^{\prime} \\
& \tau^{\prime} \geq 0 \\
& \lambda_{j} \geq 0 \text {, } \\
& r=1, \ldots, s, \\
& j=1, \ldots, n,
\end{aligned}
$$




$$
\begin{array}{ll}
\delta_{r_{o}}^{\prime} \geq 0, & r=1, \ldots, s, \\
0 \leq \alpha \leq \theta_{o}^{*} . &
\end{array}
$$

The feasible set of model (3.9) is as follows:

$$
\begin{aligned}
S_{2}=\left\{\left(\alpha, \overrightarrow{\tau^{\prime}}, \vec{\lambda}, \overrightarrow{\delta_{o}^{\prime}}\right) \mid \vec{\lambda} X \leq x_{o}+\alpha \vec{w} x_{o}, \vec{\lambda} Y \geq y_{o}-\overrightarrow{\delta_{o}^{\prime}} y_{o}, \overrightarrow{1} \cdot \vec{\lambda}=1, \overrightarrow{1} \cdot \overrightarrow{\delta_{o}^{\prime}}=1, \vec{\lambda} \in R_{\geq}^{n}, \overrightarrow{\delta_{o}^{\prime}} \in\right. \\
\left.\quad R_{\geq}^{s}, \alpha \leq \theta_{o}^{\prime}, \overrightarrow{\tau^{\prime}} \leq \overrightarrow{\delta_{o}^{\prime}}\right\} .
\end{aligned}
$$

where $\overrightarrow{\tau^{\prime}}=\left(\tau^{\prime}, \ldots, \tau^{\prime}\right)$.

Therefore, there are two the following cases:

(1) If $S_{2}=\emptyset$, then there exists no output direction vector along which the components of the output vector of $\mathrm{DMU}_{o}$ decrease. Therefore, $\mathrm{DMU}_{o}$ is not directionally congested from the right along $\vec{w}=\left(w_{1}, w_{2}, \ldots, w_{m}\right)$ and it is not directionally congested along $\vec{w}$.

(2) Let $S_{2} \neq \emptyset$ and $\left(\alpha^{*}, \tau^{\prime *}, \overrightarrow{\lambda^{*}}, \overrightarrow{\delta_{o}^{\prime}}\right)$ be an optimal solution for model (3.9). Therefore $\left(\delta_{1 o}^{\prime *}, \delta_{2 o}^{\prime *}, \ldots, \delta_{s o}^{*}\right)$ is the output direction vector along which the components of the output vector of $\mathrm{DMU}_{o}$ have the maximum possible decrease. This means that $\mathrm{DMU}_{o}$ is directionally congested from the right along $\vec{w}=\left(w_{1}, w_{2}, \ldots, w_{m}\right)$.

Now, we show the relationship between our defined directionally congestion and the classical definitions for the strong and weak congestion.

Theorem 3.3. If $\mathrm{DMU}_{o}$ is directionally congested from the left along the input direction vector $\vec{w}=$ $\left(w_{1}, w_{2}, \ldots, w_{m}\right)$, it is weakly congested.

Proof. If $\mathrm{DMU}_{o}$ is directionally congested from the left along $\vec{w}=\left(w_{1}, w_{2}, \ldots, w_{m}\right)$ then the components of the input vector of $\mathrm{DMU}_{o}$ decrease along $\vec{w}$ and there exists an output direction vector $\overrightarrow{\delta_{o}}=\left(\delta_{1 o}, \delta_{2 o}, \ldots, \delta_{s o}\right)$ along which the components of the output vector of $\mathrm{DMU}_{o}$ are increased. Since, some components of $\vec{w}=$ $\left(w_{1}, w_{2}, \ldots, w_{m}\right)$ and $\overrightarrow{\delta_{o}}=\left(\delta_{1 o}, \delta_{2 o}, \ldots, \delta_{s o}\right)$ may be zero, there exists at least one activity in $P_{\text {convex }}$ that uses less resources in some inputs to make more products in some outputs. In other words, $\mathrm{DMU}_{o}$ is weakly congested.

Theorem 3.4. Suppose that $\mathrm{DMU}_{o}$ is directionally congested from the left along the input direction vector $\vec{w}=\left(w_{1}, w_{2}, \ldots, w_{m}\right)>0$. If the components of the output direction vector, obtained from system (3.2) or model (3.6), are positive, i.e., $\overrightarrow{\delta_{o}}=\left(\delta_{1 o}, \delta_{2 o}, \ldots, \delta_{\text {so }}\right)>0$, then $\mathrm{DMU}_{o}$ is strongly congested.

Proof. Since $\vec{w}=\left(w_{1}, w_{2}, \ldots, w_{m}\right)>0$ and $\mathrm{DMU}_{o}$ is directionally congested from the left, therefore, all input components are reduced along $\vec{w}$. Suppose that the components of the output direction vector, obtained by Algorithms I and II, are positive, i.e., $\overrightarrow{\delta_{o}}=\left(\delta_{1 o}, \delta_{2 o}, \ldots, \delta_{s o}\right)>0$. This means that, all output components are increased along $\overrightarrow{\delta_{o}}$. In other words, by decreasing all input components of $\mathrm{DMU}_{o}$ along $\vec{w}$, all output components of this unit are increased along the output direction vector $\overrightarrow{\delta_{o}}=\left(\delta_{1 o}, \delta_{2 o}, \ldots, \delta_{s o}\right)$. Therefore, $\mathrm{DMU}_{o}$ is strongly congested.

\subsection{The second scenario}

Now, we consider the scenario where none of input or output directions are specified. At the first step, we try to find an input direction vector $\overrightarrow{w_{o}}=\left(w_{1 o}, w_{2 o}, \ldots, w_{m o}\right)$ and an output direction vector $\overrightarrow{\delta_{o}}=\left(\delta_{1 o}, \delta_{2 o}, \ldots, \delta_{s o}\right)$ such that the input components of $\mathrm{DMU}_{o}$ decrease along $\vec{w}_{o}$, and the output components of $\mathrm{DMU}_{o}$ increase along $\overrightarrow{\delta_{o}}$. If such vectors exist, then $\mathrm{DMU}_{o}$ is directionally congested from the left. At the second step, we try to find an input direction vector $\overrightarrow{w_{o}^{\prime}}=\left(w_{1 o}^{\prime}, w_{2 o}^{\prime}, \ldots, w_{m o}^{\prime}\right)$ and an output direction vector $\overrightarrow{\delta_{o}^{\prime}}=\left(\delta_{1 o}^{\prime}, \delta_{2 o}^{\prime}, \ldots, \delta_{s o}^{\prime}\right)$ such that the input components of $\mathrm{DMU}_{o}$ increase along $\overrightarrow{w_{o}^{\prime}}$ and the output components of $\mathrm{DMU}_{o}$ decrease along $\overrightarrow{\delta_{o}^{\prime}}$. If such vectors exist, then $\mathrm{DMU}_{o}$ is directionally congested from the right. Finally, if $\mathrm{DMU}_{o}$ is directionally congested from both sides, then this unit is directionally congested. The following algorithm recognizes the directional congestion. 


\section{Algorithm III}

Step 1. Solve system (3.11):

$$
\begin{array}{ll}
\sum_{j=1}^{n} \lambda_{j} x_{i j}=x_{i o}-w_{i o} x_{i o}, & i=1, \ldots, m, \\
\sum_{j=1}^{n} \lambda_{j} y_{r j} \geq y_{r o}+\delta_{r o} y_{r o}, & r=1, \ldots, s, \\
\sum_{j=1}^{n} \lambda_{j}=1, & \\
\sum_{r=1}^{s} \delta_{r o}=1, & \\
\sum_{i=1}^{m} w_{i o}=1, & \\
\lambda_{j} \geq 0, & \\
\delta_{r o} \geq 0, & \\
w_{i o} \geq 0, & r=1, \ldots, n, \ldots, s, \\
& \\
& \\
&
\end{array}
$$

If system (3.11) has no solution, then $\mathrm{DMU}_{o}$ is not directionally congested from the left and consequently it is not directionally congested along any input and output directions. Otherwise, suppose that $\vec{w}_{o}=\left(w_{1 o}, w_{2 o}, \ldots, w_{m o}\right)$ and $\overrightarrow{\delta_{o}}=\left(\delta_{1 o}, \delta_{2 o}, \ldots, \delta_{s o}\right)$ are the solutions of system $(3.11)$. Therefore, the components of the output vector of $\mathrm{DMU}_{o}$ can not decrease along $\overrightarrow{\delta_{o}}$ and the components of the input vector of $\mathrm{DMU}_{o}$ can not increase along $\vec{w}_{o}$. In other words, there exists an activity belongings to $P_{\text {convex }}$ that uses less resources in one or more inputs to make more products in one or more outputs. Therefore $\mathrm{DMU}_{o}$ is directionally congested from the left along the directions $\overrightarrow{w_{o}}=\left(w_{1 o}, w_{2 o}, \ldots, w_{m o}\right)$ and $\overrightarrow{\delta_{o}}=\left(\delta_{1 o}, \delta_{2 o}, \ldots, \delta_{s o}\right)$

Step 2. Solve system (3.12):

$$
\begin{array}{ll}
\sum_{j=1}^{n} \lambda_{j} x_{i j}=x_{i o}+w_{i o}^{\prime} x_{i o}, & i=1, \ldots, m, \\
\sum_{j=1}^{n} \lambda_{j} y_{r j} \geq y_{r o}-\delta_{r o}^{\prime} y_{r o}, & r=1, \ldots, s, \\
\sum_{j=1}^{n} \lambda_{j}=1, & \\
\sum_{r=1}^{s} \delta_{r o}^{\prime}=1, & \\
\sum_{i=1}^{m} w_{i o}^{\prime}=1, & \\
\lambda_{j} \geq 0, & j=1, \ldots, n, \\
\delta_{r o}^{\prime} \geq 0, & r=1, \ldots, s, \\
w_{i o}^{\prime} \geq 0, & i=1, \ldots, m .
\end{array}
$$


If system (3.12) has no solution, then $\mathrm{DMU}_{o}$ is not directionally congested from the right and consequently it is not directionally congested along any input and output directions. Otherwise, suppose that $\overrightarrow{w_{o}^{\prime}}=\left(w_{1 o}^{\prime}, w_{2 o}^{\prime}, \ldots, w_{m o}^{\prime}\right)$ and $\overrightarrow{\delta_{o}^{\prime}}=\left(\delta_{1 o}^{\prime}, \delta_{2 o}^{\prime}, \ldots, \delta_{s o}^{\prime}\right)$ are the solutions of system (3.12). Therefore, the components of the output vector of $\mathrm{DMU}_{o}$ can not increase along $\overrightarrow{\delta_{o}^{\prime}}$ and the components of the input vector of $\mathrm{DMU}_{o}$ can not decrease along $\overrightarrow{w_{o}^{\prime}}$. Therefore $\mathrm{DMU}_{o}$ is directionally congested from the right along the directions $\overrightarrow{w_{o}^{\prime}}=\left(w_{1 o}^{\prime}, w_{2 o}^{\prime}, \ldots, w_{m o}^{\prime}\right)$ and $\overrightarrow{\delta_{o}^{\prime}}=\left(\delta_{1 o}^{\prime}, \delta_{2 o}^{\prime}, \ldots, \delta_{s o}^{\prime}\right)$. If $\mathrm{DMU}_{o}$ is directionally congested on both sides then this unit is directionally congested.

In the next section, we illustrate our proposed algorithm for the various scenarios in the case studies from Tone and Sahoo [21] and Yang [28].

\section{NumERICAL EXAMPLES}

This section uses two numerical examples taken from DEA literature to compare our proposed method for identifying the directional congestion of units with the existing methods.

Example 4.1. In this example, the results of applying the proposed approach to the data set of chain stores in Japan for a period of 27 years from 1957 trough 2001, reported in Tone and Sahoo [21], are presented. This dataset has two inputs, the number of stores $\left(x_{1}\right)$ and the total area of stores $\left(x_{2}\right)$ (unit: $1000 \mathrm{~m}^{2}$ ) and one output, annual sales $y_{1}$ (unit: hundred million yen). The input and output data are reported in Table 1.

TABLE 1. Chain stores data set.

\begin{tabular}{lllll}
\hline \hline DMU & $x_{1}$ & $x_{2}$ & $y_{1}$ & Congestion status \\
\hline 1 & 2412 & 5480 & 41091 & - \\
2 & 3163 & 6233 & 48367 & Weak \\
3 & 3350 & 6798 & 56000 & Weak \\
4 & 3371 & 7274 & 60940 & - \\
5 & 3778 & 7992 & 69046 & - \\
6 & 4020 & 8500 & 77347 & - \\
7 & 5029 & 9246 & 85805 & - \\
8 & 5164 & 9639 & 90433 & - \\
9 & 5285 & 9981 & 95640 & - \\
10 & 5618 & 10276 & 100257 & - \\
11 & 5981 & 10521 & 105944 & Weak \\
12 & 6217 & 10766 & 109857 & Weak \\
13 & 6455 & 11144 & 116114 & Weak \\
14 & 6674 & 11418 & 125404 & Weak \\
15 & 6829 & 11717 & 131862 & Weak \\
16 & 6995 & 11987 & 140817 & - \\
17 & 7338 & 12463 & 150583 & - \\
18 & 7946 & 13426 & 152943 & Weak \\
19 & 8236 & 14147 & 155128 & Weak \\
20 & 7722 & 15014 & 158714 & Weak \\
21 & 7727 & 15022 & 161739 & Weak \\
22 & 7822 & 16191 & 169786 & - \\
24 & 7531 & 16969 & 167195 & Strong \\
24 & 7201 & 17627 & 167187 & - \\
25 & 7281 & 18364 & 165480 & Strong \\
26 & 7053 & 19698 & 162847 & Weak \\
27 & 6067 & 16176 & 154671 & - \\
\hline & & & & \\
\hline
\end{tabular}


TABLE 2. The directional congestion of units along $\overrightarrow{w_{1}}=(0.3,0.7)$.

\begin{tabular}{lllllll}
\hline \hline DMU & $\theta_{o}^{*}$ & $\overrightarrow{\delta_{o}}$ & LHC & $\theta_{o}^{\prime *}$ & $\overrightarrow{\delta_{o}^{\prime}}$ & RHC \\
\hline 1 & 0.000 & - & No & 0.000 & - & No \\
2 & 0.000 & - & No & 0.000 & - & No \\
3 & 0.088 & - & No & 0.000 & - & No \\
4 & 0.204 & - & No & 0.574 & - & No \\
5 & 0.217 & - & No & 0.788 & - & No \\
6 & 0.240 & - & No & 0.845 & - & No \\
7 & 0.061 & - & No & 1.474 & - & No \\
8 & 0.101 & - & No & 1.352 & - & No \\
9 & 0.132 & - & No & 1.251 & - & No \\
10 & 0.089 & - & No & 1.073 & - & No \\
11 & 0.027 & - & No & 0.905 & - & No \\
12 & 0.005 & - & No & 0.795 & - & No \\
13 & 0.010 & - & No & 0.678 & - & No \\
14 & 0.000 & - & No & 0.584 & - & No \\
15 & 0.011 & - & No & 0.512 & - & No \\
16 & 0.013 & - & No & 0.442 & - & No \\
17 & 0.000 & - & No & 0.312 & - & No \\
18 & 0.000 & - & No & 0.102 & - & No \\
19 & 0.000 & - & No & 0.000 & - & No \\
20 & 0.284 & - & No & 0.076 & 1.000 & Yes \\
21 & 0.284 & - & No & 0.075 & 1.000 & Yes \\
22 & 0.388 & - & No & 0.000 & - & No \\
23 & 0.502 & 1.000 & Yes & 0.025 & 1.000 & Yes \\
24 & 0.594 & - & No & 0.063 & 1.000 & Yes \\
25 & 0.628 & 1.000 & Yes & 0.013 & 1.000 & Yes \\
26 & 0.724 & - & No & 0.000 & - & No \\
27 & 0.655 & - & No & 0.087 & 1.000 & Yes \\
\hline
\end{tabular}

The last column of Table 1 shows the strongly and weakly congested units obtained by applying TS method.

We use model (2.4) and equation (2.5) to determine the strongly efficient units with respect to $p_{\text {convex }}$ and the projection point of each DMU on the strongly efficient frontier of $p_{\text {convex }}$, respectively, then we use the projection point of the unit to determine the directional congestion of it in our proposed methods.

In the first scenario of our proposed methods, we determine the directional congestion of units along two input direction vectors $\overrightarrow{w_{1}}=(0.3,0.7)$ and $\overrightarrow{w_{2}}=(0.9,0.1)$ by applying Algorithms I and II, respectively. The results are summarized in Tables 2 and 3 .

Table 2 reports the results of applying Algorithm I for the data set of chain stores.

Step 1. We solve model (3.1) to determine the maximum possible decrease in the components of the input vector of units along the input direction vector $\vec{w}_{1}=(0.3,0.7)$. The second column of Table 2 shows the optimal value of this model. If $\theta_{o}^{*}=0$ then $\mathrm{DMU}_{o}$ is not directionally congested from the left along the input direction vector $\vec{w}_{1}=(0.3,0.7)$. Otherwise, we go to Step 2 .

Step 2. We solve system (3.2) to obtain the output direction vector $\overrightarrow{\delta_{o}}=\left(\delta_{1 o}, \ldots, \delta_{s o}\right)$ along which the left-hand congestion occurs. The third column of Table 2 shows the output direction vectors for all units obtained by system (3.2). If system (3.2) has no solution for $\mathrm{DMU}_{o}$ then this unit is not directionally congested from the left along $\overrightarrow{w_{1}}=(0.3,0.7)$. The fourth column of Table 2 shows the status of the left-hand directional congestion of units (LHC).

Step 3. We solve model (3.3) to determine the maximum possible increase in the components of the input vector of $\mathrm{DMU}_{o}$ along the input direction vector $\overrightarrow{w_{1}}=(0.3,0.7)$. The fifth column of Table 2 shows the optimal 
TABLE 3 . The directional congestion of units along $\overrightarrow{w_{2}}=(0.9,0.1)$.

\begin{tabular}{lllllll}
\hline \hline DMU & $\theta_{o}^{*}$ & $\overrightarrow{\delta_{o}}$ & LHC & $\theta_{o}^{\prime *}$ & $\overrightarrow{\delta_{o}^{\prime}}$ & RHC \\
\hline 1 & 0.000 & - & No & 0.000 & - & No \\
2 & 0.191 & - & No & 0.000 & - & No \\
3 & 0.182 & - & No & 0.000 & - & No \\
4 & 0.134 & - & No & 0.196 & 1.000 & Yes \\
5 & 0.174 & - & No & 0.202 & 1.000 & Yes \\
6 & 0.186 & - & No & 0.223 & 1.000 & Yes \\
7 & 0.329 & - & No & 0.045 & 1.000 & Yes \\
8 & 0.322 & - & No & 0.076 & 1.000 & Yes \\
9 & 0.317 & - & No & 0.102 & 1.000 & Yes \\
10 & 0.348 & - & No & 0.065 & 1.000 & Yes \\
11 & 0.382 & - & No & 0.018 & 1.000 & Yes \\
12 & 0.397 & - & No & 0.003 & 1.000 & Yes \\
13 & 0.403 & - & No & 0.007 & 1.000 & Yes \\
14 & 0.413 & - & No & 0.000 & - & No \\
15 & 0.413 & - & No & 0.007 & 1.000 & Yes \\
16 & 0.417 & - & No & 0.007 & 1.000 & Yes \\
17 & 0.427 & - & No & 0.000 & - & No \\
18 & 0.438 & - & No & 0.000 & - & No \\
19 & 0.431 & - & No & 0.000 & - & No \\
20 & 0.340 & - & No & 0.047 & 1.000 & Yes \\
21 & 0.340 & - & No & 0.046 & 1.000 & Yes \\
22 & 0.294 & - & No & 0.000 & - & No \\
23 & 0.220 & - & No & 0.017 & 1.000 & Yes \\
24 & 0.140 & - & No & 0.045 & 1.000 & Yes \\
25 & 0.111 & 1.000 & Yes & 0.009 & 1.000 & Yes \\
26 & 0.000 & - & No & 0.000 & - & No \\
27 & 0.033 & - & No & 0.302 & 1.000 & Yes \\
\hline
\end{tabular}

value of this model. If $\theta_{o}^{*}=0$ then $\mathrm{DMU}_{o}$ is not directionally congested from the right along the input direction vector $\overrightarrow{w_{1}}=(0.3,0.7)$. Otherwise, we go to Step 4 .

Step 4. We solve system (3.4) to obtain the output direction vector $\overrightarrow{\delta_{o}^{\prime}}=\left(\delta_{1 o}^{\prime}, \ldots, \delta_{s o}^{\prime}\right)$ along which the righthand congestion occurs. Column 6 of Table 2 shows the output direction vectors for all units obtained by system (3.4). If system (3.4) has no solution for $\mathrm{DMU}_{o}$ then this unit is not directionally congested from the right along $\vec{w}_{1}=(0.3,0.7)$. Column 7 shows the status of the right-hand directional congestion of units (RHC).

Now, we determine the directional congested units along $\vec{w}_{2}=(0.9,0.1)$ by applying Algorithm II. The results are summarized in Table 3. The second column of Table 3 reports the optimal value of model (3.1) with $\overrightarrow{w_{2}}=(0.9,0.1)$. The third column shows the output direction vectors $\overrightarrow{\delta_{o}}=\left(\delta_{1 o}, \ldots, \delta_{s o}\right)$ obtained by solving model (3.6). Column 4 shows the status of the left-hand directional congestion of units along $\overrightarrow{w_{2}}=(0.9,0.1)$. The fifth column reports the optimal value of model $(3.3)$ with $\overrightarrow{w_{2}}=(0.9,0.1)$. Columns 6 shows the output direction vectors $\overrightarrow{\delta_{o}^{\prime}}=\left(\delta_{1 o}^{\prime}, \ldots, \delta_{s o}^{\prime}\right)$ obtained by solving model (3.9). Finally, column 7 shows the status of the right-hand directional congestion of units along $\overrightarrow{w_{2}}=(0.9,0.1)$.

As we saw in Table $1, \mathrm{DMU}_{23}$ and $\mathrm{DMU}_{25}$ are strongly congested by TS method. Also, in our proposed method, we obtain the output direction vector $\overrightarrow{\delta_{o}}>0$, reported in Tables 2 and 3, for $o \in\{23,25\}$, so, according to Theorem 3.4, DMU23 and $\mathrm{DMU}_{25}$ are strongly congested units by our proposed methods. This shows the relationship between our proposed methods in the first scenario and the conventional methods for recognizing the congestion in the literature. 
TABLE 4. The directional congestion of units in the second scenario.

\begin{tabular}{lllllll}
\hline \hline DMU & $\overrightarrow{w_{o}}$ & $\overrightarrow{\delta_{o}}$ & LHC & $\overrightarrow{w_{o}^{\prime}}$ & $\overrightarrow{\delta_{o}^{\prime}}$ & RHC \\
\hline 1 & - & - & No & - & - & No \\
2 & $(1.0,0.0)$ & $(1.0)$ & Yes & - & - & No \\
3 & $(1.0,0.0)$ & $(1.0)$ & Yes & - & $(1.0)$ & No \\
4 & - & - & No & $(1.0,0.0)$ & $(1.0)$ & Yes \\
5 & - & - & No & $(0.0,1.0)$ & $(1.0)$ & Yes \\
6 & - & - & No & $(0.0,1.0)$ & $(1.0)$ & Yes \\
7 & - & - & No & $(0.0,1.0)$ & $(1.0)$ & Yes \\
8 & - & - & No & $(1.0,0.0)$ & $(1.0)$ & Yes \\
9 & - & - & No & $(1.0,0.0)$ & $(1.0)$ & Yes \\
10 & - & - & No & $(1.0,0.0)$ & $(1.0)$ & Yes \\
11 & $(1.0,0.0)$ & $(1.0)$ & Yes & $(0.0,1.0)$ & $(1.0)$ & Yes \\
12 & $(1.0,0.0)$ & $(1.0)$ & Yes & $(0.0,1.0)$ & $(1.0)$ & Yes \\
13 & $(1.0,0.0)$ & $(1.0)$ & Yes & $(0.0,1.0)$ & $(1.0)$ & Yes \\
14 & $(1.0,0.0)$ & $(1.0)$ & Yes & $(0.0,1.0)$ & $(1.0)$ & Yes \\
15 & $(1.0,0.0)$ & $(1.0)$ & Yes & $(0.0,1.0)$ & $(1.0)$ & Yes \\
16 & - & - & No & $(0.0,1.0)$ & $(1.0)$ & Yes \\
17 & - & - & No & $(0.0,1.0)$ & $(1.0)$ & Yes \\
18 & $(1.0,0.0)$ & $(1.0)$ & Yes & $(0.0,1.0)$ & $(1.0)$ & Yes \\
19 & $(1.0,0.0)$ & $(1.0)$ & Yes & - & - & No \\
20 & $(1.0,0.0)$ & $(1.0)$ & Yes & $(0.0,1.0)$ & $(1.0)$ & Yes \\
21 & $(1.0,0.0)$ & $(1.0)$ & Yes & $(0.0,1.0)$ & $(1.0)$ & Yes \\
22 & - & - & No & - & - & No \\
23 & $(0.2,0.8)$ & $(1.0)$ & Yes & $(0.0,1.0)$ & $(1.0)$ & Yes \\
24 & - & - & No & $(0.0,1.0)$ & $(1.0)$ & Yes \\
25 & $(0.0,1.0)$ & $(1.0)$ & Yes & $(0.0,1.0)$ & $(1.0)$ & Yes \\
26 & $(0.0,1.0)$ & $(1.0)$ & Yes & - & - & No \\
27 & - & - & No & $(0.0,1.0)$ & $(1.0)$ & Yes \\
\hline & & & & & & \\
\end{tabular}

In the second scenario of our proposed method, none of the input and output directions are available. We apply Algorithm III to recognize the directional congestion of the data set of chain stores. The results are summarized in Table 4.

Step 1. We solve system (3.11) and obtain the input direction vector $\vec{w}_{o}$ and the output direction vector $\overrightarrow{\delta_{o}}$, reported in columns 2 and 3 of Table 4, respectively. Column 4 shows the status of the left-hand directional congestion of units. As we saw in Table 1, the units 2, 3, 11, 12, 13, 14, 15, 18, 19, 20, 21, 26 are weakly congested and the units 23 and 25 are stronly congested by TS method. On the other hand, in our proposed method for the second scenario, we obtain the input direction vector $\vec{w}_{o} \geqq 0$ and the output direction vector $\overrightarrow{\delta_{o}} \geqq 0$ for all $o \in\{2,3,11,12,13,14,15,18,19,20,21,26\}$, so, according to Theorem 3.3, the units $2,3,11,12,13,14,15,18$, 19, 20, 21, 26 are weakly congested by our proposed method. Also, we obtain the input direction vector $\vec{w}_{o}>0$ and the output direction vector $\overrightarrow{\delta_{o}}>0$ for $o \in\{23,25\}$, so, according to Theorem 3.4, $\mathrm{DMU}_{23}$ and $\mathrm{DMU}_{25}$ are strongly congested units by our proposed method. This shows the relationship between our proposed method and the conventional methods for recognizing the congestion in the literature.

Step 2. We solve system (3.12) and obtain the input direction vector $\overrightarrow{w_{o}^{\prime}}$ and the output direction vector $\overrightarrow{\delta_{o}^{\prime}}$, reported in the fifth column of Table 4. Column 6 shows the status of the right-hand directional congestion of DMUs.

Example 4.2. In this example, the results of applying the proposed approach to the data set of basic research institutes in the Chinese Academy of Science (CAS) in 2010, reported in Yang [28], are presented. This dataset 
TABLE 5. The data of units in Example 4.2.

\begin{tabular}{llllllll}
\hline \hline DMU & $x_{1}$ & $x_{2}$ & $y_{1}$ & $y_{2}$ & $y_{3}$ & $y_{4}$ & Congestion status \\
\hline 1 & 252 & 117.945 & 436 & 133 & 184 & 31.558 & No \\
2 & 37 & 29.431 & 243 & 127 & 43 & 15.3041 & No \\
3 & 240 & 101.425 & 164 & 70 & 89 & 33.8365 & Weak \\
4 & 356 & 368.483 & 810 & 276 & 247 & 183.8434 & No \\
5 & 310 & 195.862 & 200 & 55 & 111 & 12.9342 & No \\
6 & 201 & 188.829 & 104 & 49 & 33 & 60.7366 & No \\
7 & 157 & 131.301 & 113 & 49 & 45 & 72.5368 & No \\
8 & 236 & 77.439 & 8 & 1 & 44 & 23.7015 & Weak \\
9 & 805 & 396.905 & 371 & 118 & 89 & 216.9885 & Strong \\
10 & 886 & 411.539 & 607 & 216 & 168 & 88.5561 & Strong \\
11 & 623 & 221.428 & 314 & 49 & 89 & 45.3597 & Strong \\
12 & 560 & 264.341 & 261 & 79 & 131 & 41.1156 & Strong \\
13 & 1344 & 900.509 & 627 & 168 & 346 & 645.4150 & No \\
14 & 508 & 344.312 & 971 & 518 & 335 & 205.4528 & No \\
15 & 380 & 161.331 & 395 & 180 & 117 & 90.0373 & Weak \\
16 & 132 & 83.972 & 229 & 138 & 62 & 32.6111 & Weak \\
\hline
\end{tabular}

has 16 units with two inputs, the full-time equivalent of full-time research staff $\left(x_{1}\right)$ and the amount of total income of each institute $\left(x_{2}\right)$ to produce four outputs, the number of international papers indexed by Web of Science from Thompson Reuters $\left(y_{1}\right)$, the number of highly-quality papers published in top research journals $\left(y_{2}\right)$, the number of graduate student enrollment in $2009\left(y_{3}\right)$ and the amount of external research funding from research contracts $\left(y_{4}\right)$. The input and output data are reported in Table 5.

We use model (2.4) and equation (2.5) to determine the strongly efficient units with respect to $p_{\text {convex }}$ and the projection point of each DMU on the strongly efficient frontier of $p_{\text {convex }}$, respectively, then we use the projection point of $\mathrm{DMU}_{o}$ to determine the directional congestion of it in our proposed methods.

In the first scenario of our proposed methods, we determine the directional congestion of units along two input direction vectors $\overrightarrow{w_{1}}=(1.7,0.3)$ and $\overrightarrow{w_{2}}=(1.1,0.9)$ by applying Algorithms I and II, respectively. The results are summarized in Tables 6 and 7 , respectively.

Table 6 reports the results of applying Algorithm I for the data set of 16 basic research institutes in CAS. The second column of Table 6 reports the optimal value of model (3.1) with $\overrightarrow{w_{1}}=(1.7,0.3)$. The third column shows the output direction vectors $\overrightarrow{\delta_{o}}=\left(\delta_{1 o}, \ldots, \delta_{s o}\right)$ obtained by solving system (3.2). Column 4 shows the status of the left-hand directional congestion of units along $\vec{w}_{1}=(1.7,0.3)$. The fifth column reports the optimal value of model $(3.3)$ with $\overrightarrow{w_{1}}=(1.7,0.3)$. Column 6 shows the output direction vectors $\overrightarrow{\delta_{o}^{\prime}}=\left(\delta_{1 o}^{\prime}, \ldots, \delta_{s o}^{\prime}\right)$ obtained by solving system (3.4). Finally, column 7 shows the status of the right-hand directional congestion of units along $\vec{w}_{1}=(1.7,0.3)$. As we saw in Table 6 , the units $8,9,10,11,12$ are strongly congested and the units $3,15,16$ are weakly congested by TS method. In our proposed method, we obtain the output direction vector $\overrightarrow{\delta_{o}}>0$ for all $o \in\{8,9,10,11,12\}$, so, according to Theorem 3.4, the units $8,9,10,11,12$ are strongly congested by our proposed method. On the other hand, we obtain the output direction vector $\overrightarrow{\delta_{o}} \geqq 0$ for all $o \in\{3,15,16\}$, so, according to Theorem 3.3, the units 3, 15, 16 are weakly congested by our proposed method. This shows the relationship between our proposed method and the conventional methods for recognizing the congestion in the literature.

Now, we determine the directional congested units along $\vec{w}_{2}=(1.1,0.9)$ by applying Algorithm II. The results are summarized in Table 7. The second column of Table 7 reports the optimal value of model (3.1) with $\overrightarrow{w_{2}}=(1.1,0.9)$. The third column shows the output direction vectors $\overrightarrow{\delta_{o}}=\left(\delta_{1 o}, \ldots, \delta_{s o}\right)$ obtained by solving model (3.6). Column 4 shows the status of the left-hand directional congestion of units along $\overrightarrow{w_{2}}=(1.1,0.9)$. The 
TABLE 6 . The directional congestion of units along $\overrightarrow{w_{1}}=(1.7,0.3)$.

\begin{tabular}{lllllll}
\hline \hline DMU & $\theta_{o}^{*}$ & $\overrightarrow{\delta_{o}}$ & LHC & $\theta_{o}{ }^{*}$ & $\overrightarrow{\delta_{o}^{\prime}}$ & RHC \\
\hline 1 & 0.333 & - & No & 0.279 & $(0.4,0.2,0.4,0.0)$ & Yes \\
2 & 0.000 & - & No & 0.000 & - & No \\
3 & 0.357 & $(0.3,0.6,0.0,0.1)$ & Yes & 0.185 & $(0.1,0.1,0.5,0.3)$ & Yes \\
4 & 0.000 & - & No & 0.976 & $(0.4,0.4,0.2,0.0)$ & Yes \\
5 & 0.247 & - & No & 0.623 & $(0.3,0.3,0.3,0.1)$ & Yes \\
6 & 0.049 & - & No & 1.431 & $(0.3,0.3,0.3,0.1)$ & Yes \\
7 & 0.105 & - & No & 1.390 & $(0.3,0.3,0.2,0.2)$ & Yes \\
8 & 0.406 & $(0.1,0.7,0.1,0.1)$ & Yes & 0.000 & - & No \\
9 & 0.337 & $(0.2,0.3,0.4,0.1)$ & Yes & 0.051 & $(0.0,0.8,0.0,0.2)$ & Yes \\
10 & 0.352 & $(0.1,0.3,0.2,0.4)$ & Yes & 0.000 & - & No \\
11 & 0.407 & $(0.1,0.6,0.1,0.2)$ & Yes & 0.000 & - & No \\
12 & 0.344 & $(0.2,0.2,0.4,0.2)$ & Yes & 0.145 & $(0.3,0.3,0.1,0.3)$ & Yes \\
13 & 0.000 & - & No & 0.000 & - & No \\
14 & 0.228 & - & No & 0.395 & $(0.2,0.3,0.2,0.3)$ & Yes \\
15 & 0.365 & $(0.3,0.5,0.2,0.0)$ & Yes & 0.158 & $(0.2,0.3,0.2,0.3)$ & Yes \\
16 & 0.218 & - & No & 0.776 & $(0.2,0.3,0.3,0.2)$ & Yes \\
\hline
\end{tabular}

TABLE 7. The directional congestion of units along $\overrightarrow{w_{2}}=(1.1,0.9)$.

\begin{tabular}{lllllll}
\hline \hline DMU & $\theta_{o}^{*}$ & $\overrightarrow{\delta_{o}}$ & LHC & $\theta_{o}^{\prime}{ }^{*}$ & $\overrightarrow{\delta_{o}^{\prime}}$ & RHC \\
\hline 1 & 0.743 & - & No & 1.748 & $(0.0,0.2,0.8,0.0)$ & Yes \\
2 & 0.000 & - & No & 30.906 & $(0.4,0.5,0.1,0.0)$ & Yes \\
3 & 0.759 & - & No & 1.576 & $(0.3,0.3,0.2,0.2)$ & Yes \\
4 & 0.000 & - & No & 0.000 & - & No \\
5 & 0.665 & - & No & 2.126 & $(0.3,0.4,0.3,0.0)$ & Yes \\
6 & 0.229 & - & No & 1.890 & $(0.3,0.3,0.3,0.1)$ & Yes \\
7 & 0.393 & - & No & 5.165 & $(0.3,0.3,0.3,0.1)$ & Yes \\
8 & 0.000 & - & No & 0.000 & - & No \\
9 & 0.769 & - & No & 0.122 & $(0.0,0.0,0.0,1.0)$ & Yes \\
10 & 0.782 & $(0.1,0.3,0.2,0.4)$ & Yes & 0.000 & - & No \\
11 & 0.814 & $(0.3,0.5,0.1,0.1)$ & Yes & 0.000 & - & No \\
12 & 0.77 & - & No & 0.427 & $(0.3,0.4,0.2,0.1)$ & Yes \\
13 & 0.681 & - & No & 0.000 & - & No \\
14 & 0.654 & - & No & 1.731 & $(0.2,0.4,0.2,0.2)$ & Yes \\
15 & 0.778 & - & No & 0.736 & $(0.3,0.3,0.1,0.3)$ & Yes \\
16 & 0.590 & - & No & 6.010 & $(0.3,0.3,0.3,0.1)$ & Yes \\
\hline
\end{tabular}

fifth column reports the optimal value of model (3.3) with $\overrightarrow{w_{2}}=(1.1,0.9)$. Columns 6 shows the output direction vectors $\overrightarrow{\delta_{o}^{\prime}}=\left(\delta_{1 o}^{\prime}, \ldots, \delta_{s o}^{\prime}\right)$ obtained by solving model (3.9). Finally, column 7 shows the status of the right-hand directional congestion of units along $\vec{w}_{2}=(1.1,0.9)$. As we see in Table 7 , we obtain the output direction vector $\overrightarrow{\delta_{o}}>0$ for $o \in\{10,11\}$, so, according to Theorem 3.4, $\mathrm{DMU}_{10}$ and $\mathrm{DMU}_{11}$ are strongly congested units by our proposed method and TS method.

In the second scenario of our proposed method, none of the input and output directions are available. We apply algorithm III to recognize the directional congestion of the data set of 16 basic institutes in CAE. The results are summarized in Table 8. 
TABLE 8. The directional congestion of 16 units in the second scenario.

\begin{tabular}{|c|c|c|c|c|c|c|}
\hline $\mathrm{DMU}$ & $\overrightarrow{w_{o}}$ & $\overrightarrow{\delta_{o}}$ & LHC & $\overrightarrow{w_{o}^{\prime}}$ & $\overrightarrow{\delta_{o}^{\prime}}$ & $\mathrm{RHC}$ \\
\hline 1 & - & - & No & $(0.4,0.6)$ & $(1.0,0.0,0.0,0.0)$ & Yes \\
\hline 2 & - & - & No & $(0.6,0.4)$ & $(0.0,1.0,0.0,0.0)$ & Yes \\
\hline 3 & $(0.5,0.5)$ & $(0.0,0.3,0.6,0.1)$ & Yes & $(0.4,0.6)$ & $(0.0,0.0,0.0,1.0)$ & Yes \\
\hline 4 & - & - & No & $(0.7,0.3)$ & $(0.4,0.6,0.0,0.0)$ & Yes \\
\hline 5 & - & - & No & $(0.5,0.5)$ & $(0.0,1.0,0.0,0.0)$ & Yes \\
\hline 6 & - & - & No & $(0.6,0.4)$ & $(0.0,1.0,0.0,0.0)$ & Yes \\
\hline 7 & - & - & No & $(0.6,0.4)$ & $(1.0,0.0,0.0,0.0)$ & Yes \\
\hline 8 & $(0.6,0.4)$ & $(0.1,0.5,0.3,0.1)$ & Yes & $(0.3,0.7)$ & - & No \\
\hline 9 & $(0.8,0.2)$ & $(0.2,0.3,0.4,0.1)$ & Yes & $(0.5,0.5)$ & $(0.0,0.0,1.0,0.0)$ & Yes \\
\hline 10 & $(0.3,0.7)$ & $(0.1,0.3,0.2,0.4)$ & Yes & $(0.3,0.7)$ & $(0.0,1.0,0.0,0.0)$ & Yes \\
\hline 11 & $(0.5,0.5)$ & $(0.1,0.6,0.1,0.2)$ & Yes & $(0.3,0.7)$ & - & No \\
\hline 12 & $(0.3,0.7)$ & $(0.2,0.4,0.2,0.2)$ & Yes & $(0.4,0.6)$ & $(0.0,1.0,0.0,0.0)$ & Yes \\
\hline 13 & - & - & No & - & - & No \\
\hline 14 & - & - & No & $(0.5,0.5)$ & $(0.3,0.7,0.0,0.0)$ & Yes \\
\hline 15 & $(1.0,0.0)$ & $(0.3,0.4,0.2,0.1)$ & Yes & $(0.4,0.4)$ & $(0.0,1.0,0.0,0.0)$ & Yes \\
\hline 16 & $(1.0,0.0)$ & $(0.3,0.4,0.2,0.1)$ & Yes & $(0.4,0.6)$ & $(0.0,1.0,0.0,0.0)$ & Yes \\
\hline
\end{tabular}

In the first step, we solve system (3.11) and obtain the input direction vector $\vec{w}_{o}$ and the output direction vector $\overrightarrow{\delta_{o}}$, reported in the second and third columns of Table 8. Column 4 shows the status of the left-hand directional congestion of units. It should be noted that, we obtain the input direction vector $\vec{w}_{o}>0$ and the output direction vector $\overrightarrow{\delta_{o}}>0$ for all $o \in\{8,9,10,11,12\}$, so, according to Theorem 3.4 , the units $8,9,10,11$ and 12 are strongly congested by our proposed method in the second scenario and TS method.

In the next step, we solve system (3.12) and obtain the input direction vector $\overrightarrow{w_{o}^{\prime}}$ and the output direction vector $\overrightarrow{\delta_{o}^{\prime}}$, reported in columns 5 and 6 . Column 7 shows the status of the right-hand directional congestion of DMUs. Therefore, the units 3, 9, 10,12, 15 and 16 are directionally congested.

It should be noted that, Yang [28] only considers the situation that both the input and output direction vectors are predetermined. In the other word, if a unit has no directional congestion along these certain directions, then we cannot obtain any information about the existence of the congestion of this unit. He set the output direction vector as $\vec{\delta}=(1,1,1,1)$ and determined the congestion of 16 basic institutes in CAE along different input direction vectors. For example, he recognized that $\mathrm{DMU}_{1}$ has no congestion along the input direction vector $\overrightarrow{w_{1}}=(1.7,0.3)$ and the output direction vector $\vec{\delta}$. Also, he showed that $\mathrm{DMU}_{15}$ has no congestion along the input direction vector $\overrightarrow{w_{2}}=(1.1,0.9)$ and the output direction vector $\vec{\delta}$. Hence, no information about the existence of congestion of $\mathrm{DMU}_{1}$ and $\mathrm{DMU}_{15}$ can be obtained by his method. To tackle this issue, the first scenario of our proposed method considers the situation that only the input direction vector is available and then determines the output direction vector along which the congestion occurs.

As we see in Tables 6, our proposed method determines the output direction vector $\overrightarrow{\delta_{1}}=(0.4,0.2,0.4,0.0)$ such that $\mathrm{DMU}_{1}$ has the right-hand congestion along $\overrightarrow{w_{1}}=(1.7,0.3)$ and $\overrightarrow{\delta_{1}}$. Also, as we see in Tables 7 , our proposed method determines the output direction vector $\overrightarrow{\delta_{15}}=(0.3,0.3,0.1,0.3)$ such that $\mathrm{DMU}_{15}$ has the right-hand congestion along $\overrightarrow{w_{2}}=(1.1,0.9)$ and $\overrightarrow{\delta_{15}}$.

Yang [28] showed that $\mathrm{DMU}_{1}$ has no congestion along the output direction vector $\vec{\delta}=(1,1,1,1)$ and 9 different input direction vectors. But, we still cannot obtain any information about the congestion status of this unit. In the second scenario of our proposed methods, both the input and output directions are not specified and our proposed method determine the input and output direction vectors along which the congestion occurs. As we see in Table 8, our proposed method determines the input direction vector $\vec{w}_{1}=(0.4,0.6)$ and the output 
direction vector $\overrightarrow{\delta_{1}}=(1.0,0.0,0.0,0.0)$ along which $\mathrm{DMU}_{1}$ has the right-hand congestion. So, our proposed methods are more powerful than the method of Yang [28].

\section{Conclusion}

The concepts of strong and weak congestion are two basic terms in the congestion literature. The definitions of strong and weak congestion have main drawbacks, for example, we do not know whether the congestion will occur or not if the DM increases or decreases the inputs and outputs dis proportionally. In this regard, Yang [28] introduced the concept of the directional congestion in DEA. He proposed methods to determine the directional congestion of units along the certain input and output directions. The main drawback of his methods is that if a unit has no congestion along the certain input and output directions, no information about the existence of the congestion along other directions can be obtained. This study considered the situation in which at least one input or output direction is not specified. Two different scenarios have been considered in this paper: (i) only the input direction vector is available. We presented two methods to recognize the directional congestion for this scenario. (ii) none of the input and output direction vectors are available. We proposed a system of inequalities to find the input and output direction vectors along which the congestion occurs in this scenario. This study addresses the relationship between our defined directional congestion and the classical definitions of strong and weak congestion. The validity of the proposed methods was demonstrated utilizing two numerical examples from literature.

\section{REFERENCES}

[1] R.D. Banker, A. Charnes and W.W. Cooper, Some models for estimating technical and scale inefficiencies in data envelopment analysis. Manage. Sci. 30 (1984) 1078-1092.

[2] P. Brockett, W. Cooper, H. Deng, L. Golden and T. Ruefli, Using DEA to identify and manage congestion. J. Prod. Anal. 22 (2004) 207-226.

[3] W.W. Cooper, R.G. Thompson and R.M. Thrall, Introduction: extensions and new developments in DEA. Ann. Oper. Res. 66 (1996) $1-45$.

[4] W.W. Cooper, L.M. Seiford and K. Tone, Data Envelopment Analysis: A comprehensive Text with Models, Applications, References and DEA-Solver Software. Kluwer Academic Publishers, Boston (2000).

[5] W.W. Cooper, H. Deng, B. Gu, S. Li and R.M. Thrall, Using DEA to improve the management of congestion in Chinese industries (1981-1997). Soc.-Econ. Plan. Sci. 35 (2001) 227-242.

[6] W.W. Cooper, B. Gu and S. Li, Comparisons and evaluations of alternative approaches to the treatment of congestion in DEA. Eur. J. Oper. Res. 132 (2001) 62-74.

[7] W.W. Cooper, L.M. Seiford and J. Zhu, Handbook on Data Envelopment Analysis. Kluwer Academic Publishers, MA, USA (2004).

[8] M. Ebrahimzade Adimi, M. Rostamy-Malkhalifeh, F.H. Lotfi and R. Mehrjoo, A new linear method to find the congestion hyperplane in DEA. Math. Sci. 13 (2019) 43-52.

[9] A. Emrouznejad and G.L. Yang, A survey and analysis of the first 40 years of scholarly literature in DEA: 1978-2016. Soc.-Econ. Plan. Sci. 61 (2018) 4-8.

[10] R. Färe and L. Svensson, Congestion of factors of production. Econometrica 48 (1980) 1745-1753.

[11] R. Fare, S. Grosskopf and C.A.K. Lovell, The measurement of Efficiency of Production. Kluwer-Nijhoff Publishing, Boston, USA (1985).

[12] A. Ghomashi and M. Abbasi, An approach to identify and evaluate congestion in data envelopment analysis. Int. J. Data Envelopment Anal. 5 (2017) 1327-1336.

[13] G.R. Jahanshahloo and M. Khodabakhshi, Suitable combination of inputs for improving outputs in DEA with determining input congestion: considering textile industry of China. Appl. Math. Comput. 151 (2004) 263-273.

[14] C. Kao, Congestion measurement and elimination under the framework of data envelopment analysis. Int. J. Prod. Econ. 123 (2010) 257-265.

[15] H. Kheirollahi, P. Hessari, V. Charles and R. Chawshini, An input relaxation model for evaluating congestion in fuzzy DEA. Croatian Oper. Res. Rev. 8 (2017) 391-408.

[16] M. Khoveyni and R. Eslami, Determining the strongly and weakly most congested firms in data envelopment analysis. International Association for Management of Technology (2017) 1-8.

[17] M. Khoveyni, R. Eslami, M. Khodabakhshi, G.R. Jahanshahloo and F. Hosseinzadeh Lotfi, Recognizing strong and weak congestion slack based in data envelopment analysis. Comput. Ind. Eng. 64 (2013) 731-738.

[18] M. Mehdiloozad, J. Zhu and B.K. Sahoo, Identification of congestion in data envelopment analysis under the occurrence of multiple projections: a reliable method capable of dealing with negative data. Eur. J. Oper. Res. 265 (2018) 644-654. 
[19] A.A. Noura, F. Hosseinzadeh Lotfi, G.R. Jahanshahloo, S. Fanati Rashidi and B.R. Parker, A new method for measuring congestion in data envelopment analysis. Soc.-Econ. Plan. Sci. 44 (2010) 240-246.

[20] T. Sueyoshi and K. Sekitani, DEA congestion and returns to scale under an occurrence of multiple optimal projections. Eur. J. Oper. Res. 194 (2009) 592-607.

[21] K. Tone and B.K. Sahoo, Degree of scale economies and congestion: a unified DEA approach. Eur. J. Oper. Res. 158 (2004) $755-772$.

[22] P. Wanke, C.P. Barros and A. Emrouznejad, A Comparison between stochastic DEA and Fuzzy DEA approaches: revisiting efficiency in Angolan banks. RAIRO: OR 25 (2018) 285-303.

[23] Q.L. Wei and H. Yan, Congestion and returns to scale in data envelopment analysis. Eur. J. Oper. Res. 153 (2004) 641-660.

[24] Q.L. Wei and H. Yan, Weak congestion in output additive data envelopment analysis. Soc.-Econ. Plan. Sci. 43 (2009) 40-54.

[25] J. Wu, Q. An, B. Xiong and Y. Chen, Congestion measurement for regional industries in China: a data envelopment analysis approach with undesirable outputs. Energy Policy 57 (2013) 7-13.

[26] F. Wu, P. Zhou and D.Q. Zhou, Measuring energy congestion in Chinese industrial sectors: a slacks-based DEA approach. Comput. Econ. 46 (2015) 479-494.

[27] F. Wu, P. Zhou and D.Q. Zhou, Does there exist energy congestion? Empirical evidence from Chinese industrial sectors. Energ. Effic. 9 (2015) 1-14.

[28] G.L. Yang, Directional Congestion in Data Envelopment Analysis. Preprint arXiv:1510.07225 (2015). 